\title{
HOPF BIFURCATION FROM INFINITY FOR PLANAR CONTROL SYSTEMS
}

\author{
JAume Llibre And EnRIQUe Ponce
}

\begin{abstract}
Symmetric piecewise linear bi-dimensional systems are very common in control engineering. They constitute a class of non-differentiable vector fields for which classical Hopf bifurcation theorems are not applicable. For such systems, sufficient and necessary conditions for bifurcation of a limit cycle from the periodic orbit at infinity are given.
\end{abstract}

\section{Introduction and statement of main results}

In this paper we are concerned with the appearance of one limit cycle from infinity for symmetric piecewise linear bi-dimensional systems. This phenomenon can be considered as a kind of generalized Hopf bifurcation from the infinity.

The systems under study are of great importance in direct control theory $[\mathbf{1}],[\mathbf{2}]$, being very common in control engineering as they include the case where the nonlinearities involved are of saturation type. They constitute a class of non-differentiable vector fields for which classical Hopf bifurcation theorems are not applicable so that specific techniques are needed in their analysis.

Thus, we consider differential systems of the form

$$
\dot{\mathbf{x}}=\mathbf{A} \mathbf{x}+\psi\left(\mathbf{c}^{T} \mathbf{x}\right) \mathbf{b}
$$

where $\mathbf{A}$ is a $2 \times 2$ real matrix and $\mathbf{x}, \mathbf{b}, \mathbf{c}$ belong to $\mathbf{R}^{2}$. Here the dot denotes derivatives with respect to the variable $s$. The nonlinearity of these systems results from the presence of the characteristic function $\psi$. A common assumption in control theory is to consider odd piecewise linear characteristic functions of the form

$$
\psi(\sigma)= \begin{cases}k_{2} \sigma-\left(k_{1}-k_{2}\right) w & \text { if } \sigma \leq-w, \\ k_{1} \sigma & \text { if }-w<\sigma<w, \\ k_{2} \sigma+\left(k_{1}-k_{2}\right) w & \text { if } w \leq \sigma .\end{cases}
$$


Note that for $k_{2}=0$ the nonlinearity $\psi$ corresponds with a saturation function, one of the most frequent nonlinearities involved in practice.

Clearly a system (1)-(2) splits into three linear systems on the regions $\mathbf{c}^{T} \mathbf{x} \leq-w,-w \leq \mathbf{c}^{T} \mathbf{x} \leq w$ and $\mathbf{c}^{T} \mathbf{x} \geq w$ and it is invariant under the symmetry $\mathbf{x} \rightarrow-\mathbf{x}$. So, system (1)-(2) is also called an odd threepiecewise linear system.

Systems (1)-(2) are linearly dominated at infinity, that is, there exists a constant matrix $\mathbf{B}=\mathbf{A}+k_{2} \mathbf{b c}^{T}$ such that

$$
\lim _{\|\mathbf{x}\| \rightarrow \infty} \frac{\left\|\mathbf{A} \mathbf{x}+\psi\left(\mathbf{c}^{T} \mathbf{x}\right) \mathbf{b}-\mathbf{B} \mathbf{x}\right\|}{\|\mathbf{x}\|}=0,
$$

and so the results of Glover [3] and He [4], which give sufficient conditions in order that a periodic orbit bifurcates from infinity, apply. However, assuming that this bifurcation occurs for a critical value of a parameter, say $\mu=0$, they do not provide any information about:

(1) whether the bifurcated periodic orbit exists for $\mu<0$ or $\mu>0$ with $|\mu|$ sufficiently small,

(2) the uniqueness of the bifurcated periodic orbit,

(3) the stability of the bifurcated periodic orbit, and

(4) an asymptotic estimate for the size of the bifurcated periodic orbit.

Our results answer the four questions just mentioned.

System (1) is called observable if the subspace $\operatorname{span}\left\{\mathbf{c}, \mathbf{A}^{T} \mathbf{c}\right\}=\mathbf{R}^{2}$. For systems (1)-(2) we define the following four parameters

$$
\begin{array}{ll}
T=\operatorname{trace}\left(\mathbf{A}+k_{1} \mathbf{b} \mathbf{c}^{T}\right), & t=\operatorname{trace}\left(\mathbf{A}+k_{2} \mathbf{b} \mathbf{c}^{T}\right), \\
D=\operatorname{det}\left(\mathbf{A}+k_{1} \mathbf{b} \mathbf{c}^{T}\right), & d=\operatorname{det}\left(\mathbf{A}+k_{2} \mathbf{b} \mathbf{c}^{T}\right) .
\end{array}
$$

Our first result is the following.

Proposition 1. Systems (1)-(2) have a periodic orbit at infinity if and only if they are observable and $4 d-t^{2}>0$.

As it will be shown, by means of a linear change of variables, observable systems (1)-(2) can be written in the form

$$
\left(\begin{array}{c}
\dot{x} \\
\dot{y}
\end{array}\right)=\left(\begin{array}{cc}
0 & -d \\
1 & t
\end{array}\right)\left(\begin{array}{l}
x \\
y
\end{array}\right)+\varphi(y)\left(\begin{array}{c}
d-D \\
T-t
\end{array}\right),
$$


where

$$
\varphi(\sigma)= \begin{cases}\operatorname{sign}(\sigma) & \text { if }|\sigma| \geq 1 \\ \sigma & \text { if }|\sigma| \leq 1 .\end{cases}
$$

We remark that (3)-(4) correspond to the observable canonical form of systems (1)-(2) where the nonlinearity $\varphi$ is further a normalized saturation. Selecting $t$ as the bifurcation parameter, we will use this form to show our main result, which is the following.

Theorem 2. For systems (1)-(2) having a periodic orbit at infinity, the following statements hold.

(a) If $T \neq 0$ then for $t=0$ a unique limit cycle bifurcates from the periodic orbit at infinity.

(b) If $T<0$ then, for $\varepsilon>0$ sufficiently small, the bifurcated limit cycle exists for $t \in(0, \varepsilon)$ and is unstable, and it does not exist for $t \in(-\varepsilon, 0)$.

(c) If $T>0$ then, for $\varepsilon>0$ sufficiently small, the bifurcated limit cycle exists for $t \in(-\varepsilon, 0)$ and is stable, and it does not exist for $t \in(0, \varepsilon)$.

(d) For their observable canonical form (3)-(4), the bifurcated limit cycle is near the ellipse of $x$-semiaxis

$$
a=\frac{T d-t D}{d} \cdot \frac{1+\exp \left(-\frac{\pi t}{\sqrt{4 d-t^{2}}}\right)}{1-\exp \left(\frac{\pi t}{\sqrt{4 d-t^{2}}}\right)},
$$

and $y$-semiaxis $b=a / \sqrt{d}$.

(e) If $T=0$ then for $t=0$ no limit cycles bifurcate from the periodic orbit at infinity.

(f) For $t \neq 0$ no limit cycles bifurcate from the periodic orbit at infinity.

Particular cases which can be studied by Theorem 2 appeared in Lum and Chua [5], Llibre and Sotomayor [6] and Llibre and Ponce [7].

Our main tool for proving Theorem 2 is the study of the first derivatives of the Poincaré map in a neighborhood of infinity. To compute these derivatives, we use a extension to piecewise smooth systems of some results of Lloyd [8].

Bifurcation of a periodic orbit from infinity has been also studied for polynomial planar vector fields, see for instance Sotomayor and Paterlini [9], Blows and Rousseau [10], and Guíñez, Sáez and Szantó [11]. 
Other papers about bifurcation of periodic orbits from infinity are due to Keith and Rand [12], Malaguti [13] and Sabatini [14], where they study the Rayleigh, Van der Pol and Liénard systems.

The paper is organized as follows. In Section 2, we summarize the results in a previous work [15] about formulas for the first derivatives of the Poincaré map for piecewise smooth systems. The proof of Proposition 1 and the use of these formulas to prove Theorem 2 are included in Section 3.

\section{First derivatives of the Poincaré map at infinity for piecewise smooth systems}

The study of the Poincaré map in a neighborhood of infinity for planar vector fields, when it is well defined, can be conveniently made by using the Bendixson transformation. This reduces the problem to a similar study in a neighborhood of the origin for the transformed system, see for instance Andronov and others [16].

To fix ideas, we consider the planar system,

$$
\begin{aligned}
\dot{x} & =f(x, y), \\
\dot{y} & =g(x, y),
\end{aligned}
$$

where $f$ and $g$ are Lipschitz functions. Through the inversion given by the Bendixson change of variables

$$
\left(\begin{array}{l}
u \\
v
\end{array}\right)=\frac{1}{x^{2}+y^{2}}\left(\begin{array}{l}
x \\
y
\end{array}\right),
$$

we can formulate an equivalent system which behaves in a neighborhood of the origin like system (5) near infinity. Using now the polar coordinates $u=r \cos \theta, v=r \sin \theta$, what of course corresponds to do from the beginning the change of variables

$$
x=\frac{\cos \theta}{r}, \quad y=\frac{\sin \theta}{r},
$$

the system becomes

$$
\begin{aligned}
& \dot{r}=-r^{2}\left[f\left(\frac{\cos \theta}{r}, \frac{\sin \theta}{r}\right) \cos \theta+g\left(\frac{\cos \theta}{r}, \frac{\sin \theta}{r}\right) \sin \theta\right], \\
& \dot{\theta}=-r\left[f\left(\frac{\cos \theta}{r}, \frac{\sin \theta}{r}\right) \sin \theta-g\left(\frac{\cos \theta}{r}, \frac{\sin \theta}{r}\right) \cos \theta\right],
\end{aligned}
$$


and we will be interested in the flow defined in the half-cylinder $\mathbf{R}^{+} \times$ $\mathbf{S}^{1}=\{(r, \theta): r \geq 0, \theta \in[0,2 \pi)\}$.

System (6) has in most cases no sense for $r=0$, but this difficulty can be normally overcomed by a time reparametrization, and typically it suffices to multiply its vector field by an adequate power of $r$. Also note that, after extending continuously the flow to $r=0$ if needed, the existence of a periodic orbit at infinity for system (5) is equivalent to have $r=0$ as a periodic orbit on the cylinder for system (6).

To be more precise, we assume in the sequel that system (6) can be extended to the system,

$$
\begin{aligned}
& \dot{r}=R(r, \theta), \\
& \dot{\theta}=\Theta(r, \theta),
\end{aligned}
$$

where the functions $R$ and $\Theta$ verify the following assumptions:

(A1) $R$ and $\Theta$ both are Lipschitz functions and they have period $2 \pi$ in $\theta$.

(A2) $R(0, \theta)=0$ for all $\theta$, and $\Theta(0, \theta) \neq 0$ for all $\theta$.

Note that the last assumption implies that $r=0$ is a periodic orbit of system (7) and that it has no equilibrium points in $[0, \rho) \times \mathbf{S}^{1}$ for some $\rho$ sufficiently small. This represents a sufficient and necessary condition for system (5) to have a periodic orbit at infinity.

Since $\Theta \neq 0$ in a neighborhood of $r=0$, we can regard (7) as the first order equation

$$
\frac{d r}{d \theta}=\mathcal{S}(r, \theta)=\frac{R(r, \theta)}{\Theta(r, \theta)},
$$

where $r \in[0, \rho), \theta \in \mathbf{S}^{1}$. For $\xi \in[0, \rho)$, we denote with $r(\theta, \xi)$ the solution of $(8)$ satisfying $r(0, \xi)=\xi$. We consider the Poincaré map $\xi \mapsto h(\xi)=r(2 \pi, \xi)$ and assume that $h(\xi)$ is defined in $[0, \rho)$. Of course, $h(0)=0$. As it is well-known, $h$ is monotonically increasing on its domain of definition and every solution of $h(\xi)-\xi=0$ corresponds with a periodic orbit of (8) and consequently of (7), (6) and (5).

By studying the behaviour of the first derivatives of $h$, we can deduce whether additional solutions of the equation $h(\xi)-\xi=0$ bifurcate from the solution $\xi=0$. New solutions, if any, will correspond with periodic orbits of system (5) bifurcating from the periodic orbit at infinity.

When $R$ and $\Theta$, and so $\mathcal{S}$, are sufficiently smooth, these first derivatives can be computed following Lloyd [8]. However, the lack of smoothness of 
differential systems (1)-(2) requires additional caveats. In what follows, we consider the case when the corresponding vector field $\mathcal{S}(r, \theta)$ is only piecewise differentiable and we are interested in computing the first and second derivatives of a generic Poincaré map.

We assume a domain $\mathcal{D}=\left\{(r, \theta) \in[0, \rho) \times\left[\theta_{1}, \theta_{2}\right]\right\}$ which is halved by the graph of a $\mathcal{C}^{1}$-function $\bar{\theta}:[0, \rho) \mapsto\left[\theta_{1}, \theta_{2}\right]$ in the two pieces $\mathcal{D}_{1}=\left\{(r, \theta): r \in[0, \rho), \theta \in\left[\theta_{1}, \bar{\theta}(r)\right]\right\}$ and $\mathcal{D}_{2}=\{(r, \theta): r \in[0, \rho), \theta \in$ $\left.\left[\bar{\theta}(r), \theta_{2}\right]\right\}$, so that

$$
\mathcal{S}(r, \theta)= \begin{cases}\mathcal{S}_{1}(r, \theta) & \text { for }(r, \theta) \in \mathcal{D}_{1}, \\ \mathcal{S}_{2}(r, \theta) & \text { for }(r, \theta) \in \mathcal{D}_{2} \backslash \mathcal{D}_{1}\end{cases}
$$

where both $\mathcal{S}_{1}: \mathcal{D}_{1} \mapsto \mathbf{R}$ and $\mathcal{S}_{2}: \mathcal{D}_{2} \mapsto \mathbf{R}$ are smooth, and $\mathcal{S}(0, \theta)=0$, for all $\theta \in\left[\theta_{1}, \theta_{2}\right]$.

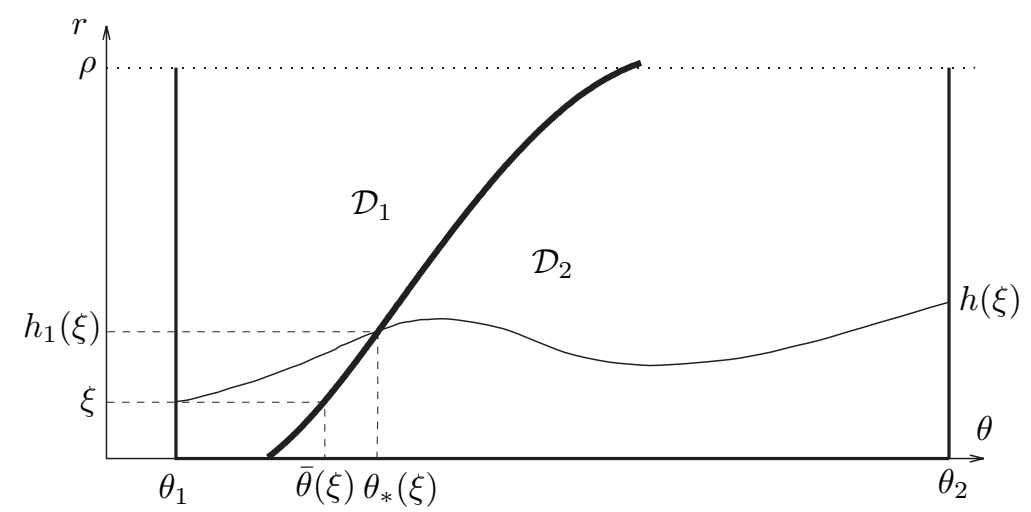

Figure 1. Scheme of the situation considered in this section.

We suppose that the corresponding equation (8) with $\mathcal{S}$ given in (9) has for all $\xi \in[0, \rho)$ a continuous solution $r(\theta, \xi)$ defined in $\theta_{1} \leq \theta \leq \theta_{2}$ which verifies $r\left(\theta_{1}, \xi\right)=\xi$. We also assume for all $\xi \in[0, \rho)$ a transversality condition

$$
\mathcal{S}_{1}(\xi, \bar{\theta}(\xi)) \cdot \frac{d \bar{\theta}}{d r}(\xi) \neq 1
$$

on the curve $\bar{\theta}([0, \rho))$, and the existence of a $\mathcal{C}^{1}$ crossing phase function,

$$
\theta_{*}:[0, \rho) \mapsto\left[\theta_{1}, \theta_{2}\right] \text { with } \theta_{*}(\xi)=\bar{\theta}\left(r\left(\theta_{*}(\xi), \xi\right)\right),
$$


which permits to define the intermediate Poincaré map

$$
\xi \mapsto h_{1}(\xi)=r\left(\theta_{*}(\xi), \xi\right) .
$$

See Figure 1. The following result has been proved in a previous work [15].

Proposition 3. Under the previous assumptions, for $\xi \in[0, \rho)$ and $\theta \in\left[\theta_{1}, \theta_{2}\right]$ we define the functions:

$$
\begin{aligned}
E\left(\xi, \theta_{1}, \theta\right) & =\exp \left(\int_{\theta_{1}}^{\theta} \frac{\partial \mathcal{S}}{\partial r}(r(\phi, \xi), \phi) d \phi\right), \\
D(\xi, \theta) & =E\left(\xi, \theta_{1}, \theta\right) \cdot \frac{\partial^{2} \mathcal{S}}{\partial r^{2}}(r(\theta, \xi), \theta) .
\end{aligned}
$$

Then the Poincaré map $\xi \mapsto h(\xi)=r\left(\theta_{2}, \xi\right)$ verifies:

$$
\begin{aligned}
h^{\prime}(\xi) & =\frac{1-\mathcal{S}_{2}\left(h_{1}(\xi), \bar{\theta}\left(h_{1}(\xi)\right)\right) \cdot \bar{\theta}^{\prime}\left(h_{1}(\xi)\right)}{1-\mathcal{S}_{1}\left(h_{1}(\xi), \bar{\theta}\left(h_{1}(\xi)\right)\right) \cdot \bar{\theta}^{\prime}\left(h_{1}(\xi)\right)} E\left(\xi, \theta_{1}, \theta_{2}\right), \\
h^{\prime}(0) & =E\left(0, \theta_{1}, \theta_{2}\right)
\end{aligned}
$$

If furthermore the vector field $\mathcal{S}$ given in (9) is continuous, we have

$$
\begin{aligned}
h^{\prime \prime}(\xi)= & E\left(\xi, \theta_{1}, \theta_{2}\right) \cdot \int_{\theta_{1}}^{\theta_{2}} D(\xi, \theta) d \theta \\
& +E\left(\xi, \theta_{1}, \theta_{2}\right) \frac{E\left(\xi, \theta_{1}, \bar{\theta}\left(h_{1}(\xi)\right)\right) \cdot \bar{\theta}^{\prime}\left(h_{1}(\xi)\right)}{1-\mathcal{S}_{1}\left(h_{1}(\xi), \bar{\theta}\left(h_{1}(\xi)\right)\right) \cdot \bar{\theta}^{\prime}\left(h_{1}(\xi)\right)} \\
& \cdot\left(\frac{\partial \mathcal{S}_{1}}{\partial r}\left(h_{1}(\xi), \bar{\theta}\left(h_{1}(\xi)\right)\right)-\frac{\partial \mathcal{S}_{2}}{\partial r}\left(h_{1}(\xi), \bar{\theta}\left(h_{1}(\xi)\right)\right)\right),
\end{aligned}
$$

and

$$
\text { (14) } \begin{aligned}
h^{\prime \prime}(0)= & E\left(0, \theta_{1}, \theta_{2}\right) \cdot\left[\int_{\theta_{1}}^{\theta_{2}} D(0, \theta) d \theta\right. \\
& \left.+E\left(0, \theta_{1}, \bar{\theta}(0)\right) \cdot \bar{\theta}^{\prime}(0)\left(\frac{\partial \mathcal{S}_{1}}{\partial r}(0, \bar{\theta}(0))-\frac{\partial \mathcal{S}_{2}}{\partial r}(0, \bar{\theta}(0))\right)\right] .
\end{aligned}
$$

We remark that these formulae are in general different of those obtained by Lloyd [8], and of course both coincide if the vector field $\mathcal{S}$ turns out to be sufficiently differentiable. Proposition 3 will be used in the next section to prove Theorem 2 . 


\section{Proof of the main results}

We start by showing two intermediate results which will be used in the proof of Proposition 1.

Lemma 4. If a system (1)-(2) has a periodic orbit at infinity then it is observable.

Proof: Suppose that a system (1)-(2) is not observable. Then, there exists a vector $\mathbf{v} \neq \mathbf{0}$ such that $\left(\mathbf{c}, \mathbf{A}^{T} \mathbf{c}\right)^{T} \mathbf{v}=\mathbf{0}$, and so both $\mathbf{c}^{T} \mathbf{v}=0$ and $\mathbf{c}^{T} \mathbf{A v}=0$. Then $\mathbf{v}$ is an eigenvector of the matrix $\mathbf{A}$, because if $\mathbf{A} \mathbf{v} \neq \lambda \mathbf{v}$ for all $\lambda \in \mathbf{R}$ we get the contradiction $0=\mathbf{c}^{T} \mathbf{A} \mathbf{v} \neq \lambda \mathbf{c}^{T} \mathbf{v}=0$. Since $\left(\mathbf{A}+k_{1} \mathbf{b} \mathbf{c}^{T}\right) \mathbf{v}=\mathbf{A} \mathbf{v}$, the vector $\mathbf{v}$ is also an eigenvector of the matrix $\mathbf{A}+k_{1} \mathbf{b} \mathbf{c}^{T}$. We note that system (1)-(2) is $\dot{\mathbf{x}}=\left(\mathbf{A}+k_{1} \mathbf{b} \mathbf{c}^{T}\right) \mathbf{x}$, for $\left|\mathbf{c}^{T} \mathbf{x}\right| \leq u$. So that the straightline $\mathbf{c}^{T} \mathbf{x}=0$ is invariant under its flow. Consequently, we have a symmetric pair of critical points in the equator of the Poincaré sphere and the conclusion follows.

Lemma 5. Observable systems (1)-(2) can be written by means of a linear change of variables in the form given in (3)-(4).

Proof: If we make in (1)-(2) the changes $w \mathbf{y}=\mathbf{x}, \overline{\mathbf{A}}=\mathbf{A}+k_{2} \mathbf{b c}^{T}$ and $\overline{\mathbf{b}}=\left(k_{1}-k_{2}\right) \mathbf{b}$, we obtain the system

$$
\dot{\mathbf{y}}=\overline{\mathbf{A}} \mathbf{y}+\varphi\left(\mathbf{c}^{T} \mathbf{y}\right) \bar{b},
$$

where $\varphi$ is given in (4). This transformation preserves the hypothesis of observability because this hypothesis is equivalent to $\operatorname{det}\left(\mathbf{c}, \mathbf{A}^{T} \mathbf{c}\right) \neq 0$, and

$$
\operatorname{det}\left(\mathbf{c}, \overline{\mathbf{A}}^{T} \mathbf{c}\right)=\operatorname{det}\left(\mathbf{c}, \mathbf{A}^{T} \mathbf{c}+\left(k_{2} \mathbf{b}^{T} \mathbf{c}\right) c\right)=\operatorname{det}\left(\mathbf{c}, \mathbf{A}^{T} \mathbf{c}\right) .
$$

Now, from (15) and by means of another linear change of variables, we can pass to the observable canonical form (see Theorem 7-2 of Chen $[\mathbf{1 7}])$ :

$$
\left(\begin{array}{l}
\dot{x} \\
\dot{y}
\end{array}\right)=\left(\begin{array}{cc}
0 & -d \\
1 & t
\end{array}\right)\left(\begin{array}{l}
x \\
y
\end{array}\right)+\varphi(y)\left(\begin{array}{l}
b_{1} \\
b_{2}
\end{array}\right) .
$$

We remark that the quoted theorem guarantees the existence of an invertible matrix $\mathbf{T}$ such that

$$
\mathbf{c}^{T} \mathbf{T}=(0,1), \quad\left(\begin{array}{cc}
0 & -d \\
1 & t
\end{array}\right)=\mathbf{T}^{-1} \overline{\mathbf{A}} \mathbf{T}, \text { and }\left(\begin{array}{l}
b_{1} \\
b_{2}
\end{array}\right)=\mathbf{T}^{-1} \overline{\mathbf{b}}
$$


Comparing now the vector field near the origin of system (16) with that of (1)-(2), we have $b_{1}-d=-D$ and $b_{2}+t=T$, and the lemma follows.

In the rest of the paper, we use for system (3)-(4) the notation of (16), where

$$
b_{1}=d-D, \text { and } b_{2}=T-t,
$$

and we remark that it is formed by the following linear systems

$$
\begin{array}{ll}
\left(\begin{array}{l}
\dot{x} \\
\dot{y}
\end{array}\right)=\left(\begin{array}{cc}
0 & -d \\
1 & t
\end{array}\right)\left(\begin{array}{l}
x \\
y
\end{array}\right)+\left(\begin{array}{l}
b_{1} \\
b_{2}
\end{array}\right) \quad \text { for } y \geq 1, \\
\left(\begin{array}{l}
\dot{x} \\
\dot{y}
\end{array}\right)=\left(\begin{array}{cc}
0 & b_{1}-d \\
1 & b_{2}+t
\end{array}\right)\left(\begin{array}{l}
x \\
y
\end{array}\right) \quad \text { for }|y| \leq 1, \quad \text { and } \\
\left(\begin{array}{l}
\dot{x} \\
\dot{y}
\end{array}\right)=\left(\begin{array}{cc}
0 & -d \\
1 & t
\end{array}\right)\left(\begin{array}{l}
x \\
y
\end{array}\right)-\left(\begin{array}{l}
b_{1} \\
b_{2}
\end{array}\right) \quad \text { for } y \leq-1 .
\end{array}
$$

Proof of Proposition 1: Assume first that system (1)-(2) is observable. From Lemma 5, we can pass to the equivalent formulation given in (17)(20). We define three regions on the cylinder $\mathbf{R}^{+} \times \mathbf{S}^{1}=\{(r, \theta): r \geq 0$ and $\theta \in[0,2 \pi)\}$ as follows

$$
\begin{aligned}
\mathcal{D}_{I} & =\{(r, \theta): 0 \leq \theta \leq \pi \text { and } r \leq \sin \theta\}, \\
\mathcal{D}_{I I} & =\{(r, \theta): 0 \leq \theta \leq 2 \pi \text { and } r \geq|\sin \theta|\}, \\
\mathcal{D}_{I I I} & =\{(r, \theta): \pi \leq \theta \leq 2 \pi \text { and } r \leq-\sin \theta\} .
\end{aligned}
$$

See Figure 2.

If we make the Bendixson transformation, defined in Section 2, we obtain from (6) and (18)-(20) the following three systems:

$$
\begin{aligned}
& \dot{r}=-r\left[(1-d) \sin \theta \cos \theta+t \sin ^{2} \theta+r\left(b_{1} \cos \theta+b_{2} \sin \theta\right)\right], \\
& \dot{\theta}=d \sin ^{2} \theta+\cos ^{2} \theta+t \sin \theta \cos \theta-r\left(b_{1} \sin \theta-b_{2} \cos \theta\right),
\end{aligned}
$$

where $(r, \theta) \in \mathcal{D}_{I}$,

$$
\begin{aligned}
& \dot{r}=-r\left[\left(1+b_{1}-d\right) \sin \theta \cos \theta+\left(b_{2}+t\right) \sin ^{2} \theta\right], \\
& \dot{\theta}=-\left(b_{1}-d\right) \sin ^{2} \theta+\cos ^{2} \theta-\left(b_{2}+t\right) \sin \theta \cos \theta,
\end{aligned}
$$

where $(r, \theta) \in \mathcal{D}_{I I}$, and

$$
\begin{aligned}
& \dot{r}=-r\left[(1-d) \sin \theta \cos \theta+t \sin ^{2} \theta-r\left(b_{1} \cos \theta+b_{2} \sin \theta\right)\right], \\
& \dot{\theta}=d \sin ^{2} \theta+\cos ^{2} \theta+t \sin \theta \cos \theta+r\left(b_{1} \sin \theta-b_{2} \cos \theta\right),
\end{aligned}
$$


where $(r, \theta) \in \mathcal{D}_{I I I}$. Note that the global flow on the cylinder $\mathbf{R}^{+} \times \mathbf{S}^{1}$ is invariant to the change $(r, \theta) \rightarrow(r, \theta+\pi)$, which is a consequence of the original symmetry $\mathbf{x} \leftrightarrow-\mathbf{x}$ of (1)-(2), and that it verifies assumption (A1) of Section 2.

Regarding assumption (A2), which is then a sufficient and necessary condition for having a periodic orbit at infinity, we see that $R(0, \theta)=0$ for all $\theta$ and that

$$
\Theta(0, \theta)=d \sin ^{2} \theta+\cos ^{2} \theta+t \sin \theta \cos \theta, \text { for } 0 \leq \theta \leq 2 \pi,
$$

which can be seen as a quadratic form in $(\sin \theta, \cos \theta)$ with matrix

$$
\left(\begin{array}{cc}
d & \frac{t}{2} \\
\frac{t}{2} & 1
\end{array}\right)
$$

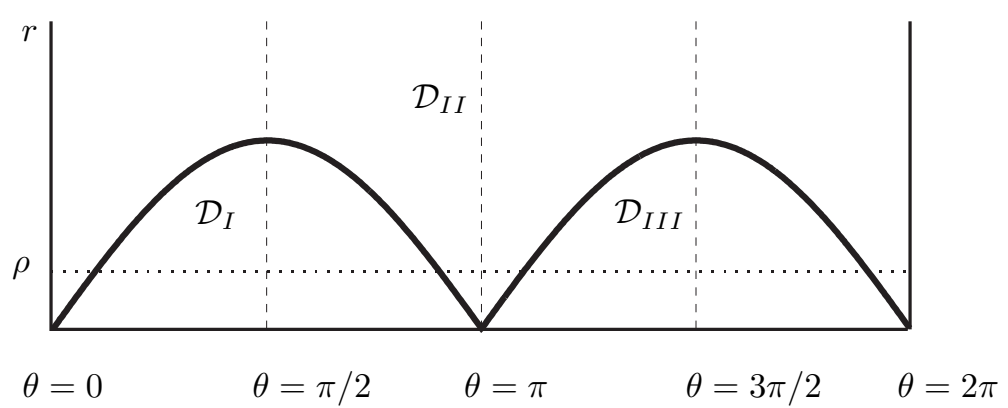

Figure 2. Different regions in $\mathbf{R}^{+} \times \mathbf{S}^{1}$ for odd three piecewise linear systems.

Hence, the condition $\Theta(0, \theta) \neq 0$ for all $\theta$ is equivalent to the above matrix be positive or negative definite. Since this is also equivalent to $4 d-t^{2}>0$, the 'if' part of the proposition follows.

To show the 'only if' part of the Proposition, it suffices to apply first Lemma 4, and to consider then the necessity of assumption (A2) of Section 2, which implies that $4 d-t^{2}>0$.

Before to give the proof of Theorem 2, we do some preparation work assuming that system (1)-(2) has a periodic orbit at infinity and so, from Proposition 1 , that it is observable and $4 d-t^{2}>0$. Thus, we can assume that $d>0$ and start from the equivalent system (16). In order to simplify the remaining computations, we can do in (16) the change

$$
x=\sqrt{d} X, \quad y=Y, \quad s=\tau / \sqrt{d},
$$


getting

$$
\begin{aligned}
& \frac{d X}{d \tau}=-Y+\bar{b}_{1} \varphi(Y) \\
& \frac{d Y}{d \tau}=X+\bar{t} Y+\bar{b}_{2} \varphi(Y)
\end{aligned}
$$

where

$$
\bar{b}_{1}=\frac{b_{1}}{d}, \quad \bar{b}_{2}=\frac{b_{2}}{\sqrt{d}} \quad \text { and } \quad \bar{t}=\frac{t}{\sqrt{d}},
$$

so that $|\bar{t}|<2$. Notice that $(25)$ is of the form (16) and so, if we omit the bar of the parameters $b_{1}, b_{2}$ and $t$, writing

$$
\left(\begin{array}{l}
\frac{d X}{d \tau} \\
\frac{d Y}{d \tau}
\end{array}\right)=\left(\begin{array}{cc}
0 & -1 \\
1 & t
\end{array}\right)\left(\begin{array}{l}
X \\
Y
\end{array}\right)+\varphi(Y)\left(\begin{array}{l}
b_{1} \\
b_{2}
\end{array}\right)
$$

it suffices now to take $d=1$ in (21)-(23) to get the vector field on the cylinder which gives the flow near the point at infinity:

$$
\mathcal{S}(r, \theta)= \begin{cases}\mathcal{S}_{I}(r, \theta)=-r \frac{t \sin ^{2} \theta+r\left(b_{1} \cos \theta+b_{2} \sin \theta\right)}{1+t \sin \theta \cos \theta-r\left(b_{1} \sin \theta-b_{2} \cos \theta\right)} & \text { in } \mathcal{D}_{I}, \\ \mathcal{S}_{I I}(r, \theta)=-r \frac{t \sin ^{2} \theta+b_{1} \sin \theta \cos \theta+b_{2} \sin ^{2} \theta}{1-b_{1} \sin ^{2} \theta-\left(b_{2}+t\right) \sin \theta \cos \theta} & \text { in } \mathcal{D}_{I I}, \\ \mathcal{S}_{I I I}(r, \theta)=-r \frac{t \sin ^{2} \theta-r\left(b_{1} \cos \theta+b_{2} \sin \theta\right)}{1+t \sin \theta \cos \theta+r\left(b_{1} \sin \theta-b_{2} \cos \theta\right)} & \text { in } \mathcal{D}_{I I I} .\end{cases}
$$

Clearly, it turns out to be piecewise smooth and Lipschitz for $|t|<2$.

As assumptions (A1) and (A2) are fulfilled, there exists $\rho>0$ such that for $\xi \in[0, \rho)$ the solution $r(\theta, \xi)$ with $r(0, \xi)=\xi$ of (8) with $\mathcal{S}(r, \theta)$ given in (28) is well defined for $0 \leq \theta \leq 2 \pi$ and such that the Poincaré map $\xi \mapsto h(\xi)=r(2 \pi, \xi)$ is analytic (we remark that $h$ is composition of five analytic functions if $\rho$ is sufficiently small, see Figure 2).

Due to the invariance of equation (8) with $\mathcal{S}$ given in (28) under the change $(r, \theta) \rightarrow(r, \theta+\pi)$, we can decompose the Poincaré map as follows,

$$
h(\xi)=h_{1}\left(h_{1}(\xi)\right) \text {, where } h_{1}(\xi)=r(\pi, \xi),
$$

and we note that $\xi_{0}$ is a fixed point of $h$ if and only if $\xi_{0}$ is a fixed point of $h_{1}$, and in that case it has the same stability for both maps because $h^{\prime}\left(\xi_{0}\right)=\left(h_{1}^{\prime}\left(\xi_{0}\right)\right)^{2}$.

In the following result we give the value of the first two derivatives of the Poincaré map $h_{1}$. 
Lemma 6. The half Poincaré map $h_{1}$ of equation (8) with $\mathcal{S}(r, \theta)$ given in (28) verifies:

$$
\begin{aligned}
h_{1}^{\prime}(0) & =\exp \left(-\frac{\pi t}{\sqrt{4-t^{2}}}\right), \\
h_{1}^{\prime \prime}(0) & =-2 h_{1}^{\prime}(0)\left(b_{1} t+b_{2}\right)\left(1+\exp \left(-\frac{\pi t}{\sqrt{4-t^{2}}}\right)\right), \\
\left.h_{1}^{\prime \prime}(0)\right|_{t=0} & =-4 b_{2} .
\end{aligned}
$$

Proof: Our main tool will be Proposition 3, and to apply it adequately we first decompose the Poincaré map $h_{1}$ in the two maps:

$\xi \mapsto h_{1,1}(\xi)=r\left(\frac{\pi}{2}, \xi\right)$ for $\xi \in[0, \rho)$ with $\rho \ll 1$,

$\eta \mapsto h_{1,2}(\eta)=r(\pi, \xi)$ for $\xi$ such that $r\left(\frac{\pi}{2}, \xi\right)=\eta$, and $\eta \in\left[0, r\left(\frac{\pi}{2}, \rho\right)\right)$,

where $r(\theta, \xi)$ is the solution such that $r(0, \xi)=\xi$.

We note that $h_{1,1}$ is the Poincaré map which applies the interval $[0, \rho)$ of the generatrix $\{\theta=0\}$ into the generatrix $\{\theta=\pi / 2\}$, see Figure 2 . Similarly $h_{1,2}$ is the Poincaré map which applies the interval $[0, r(\pi / 2, \rho))$ of the generatrix $\{\theta=\pi / 2\}$ into the generatrix $\{\theta=\pi\}$. We also have $h_{1,1}(0)=0$ and $h_{1,2}(0)=0$, because $r(\theta, 0)=0$ for all $\theta$.

It is clear that $h_{1,1}$ makes use only of the flow in the region $0 \leq \theta \leq$ $\pi / 2$, where $\mathcal{S}(r, \theta)$ is piecewise smooth, coinciding with $\mathcal{S}_{I I}(r, \theta)$ of (28) for $0 \leq \theta \leq \bar{\theta}(r)=\sin ^{-1} r$, and with $\mathcal{S}_{I}(r, \theta)$ of $(28)$ for $\bar{\theta}(r) \leq \theta \leq \pi / 2$.

In order to apply Proposition 3, we must check the transversality condition (10) for $\mathcal{S}_{I I}(r, \theta)$. It comes from a continuity argument by seeing that $\bar{\theta}^{\prime}(0)=1$ and $\mathcal{S}_{I I}(0,0)=0$.

From (12),

$$
h_{1,1}^{\prime}(0)=E\left(0,0, \frac{\pi}{2}\right)
$$

where

$$
E(0,0, \theta)=\exp \left(\int_{0}^{\theta} \frac{\partial \mathcal{S}_{I}}{\partial r}(0, \phi) d \phi\right)
$$

because the value of $\frac{\partial \mathcal{S}_{I I}}{\partial r}$ does not contribute to the integral.

Elementary calculations show that

$$
\begin{aligned}
\frac{\partial \mathcal{S}_{I}}{\partial r}(0, \theta) & =-\frac{t \sin ^{2} \theta}{1+t \sin \theta \cos \theta} \\
\frac{\partial^{2} \mathcal{S}_{I}}{\partial r^{2}}(0, \theta) & =-2 \frac{b_{1}(\cos \theta+t \sin \theta)+b_{2} \sin \theta}{(1+t \sin \theta \cos \theta)^{2}}
\end{aligned}
$$


and the expression (14) reduces to

$$
h_{1,1}^{\prime \prime}(0)=E\left(0,0, \frac{\pi}{2}\right) \int_{0}^{\frac{\pi}{2}} D(0, \theta) d \theta,
$$

because $\frac{\partial \mathcal{S}_{I}}{\partial r}(0,0)=\frac{\partial \mathcal{S}_{I I}}{\partial r}(0,0)=0$ and so the second term in (14) is zero. We also have

$$
D(0, \theta)=E(0,0, \theta) \frac{\partial^{2} \mathcal{S}_{I}}{\partial r^{2}}(0, \theta),
$$

because the integration can be made completely in $\mathcal{D}_{I}$. In short, we get

$$
h_{1,1}^{\prime \prime}(0)=E\left(0,0, \frac{\pi}{2}\right) \int_{0}^{\frac{\pi}{2}} \frac{\partial^{2} \mathcal{S}_{I}}{\partial r^{2}}(0, \theta) E(0,0, \theta) d \theta .
$$

Now we apply Proposition 3 to $h_{1,2}$, and note that all the computations are similar but $\bar{\theta}(r)=\pi-\sin ^{-1} r, \theta_{1}=\pi / 2$, and $\theta_{2}=\pi$. So, we obtain

$$
\begin{aligned}
& h_{1,2}^{\prime}(0)=E\left(0, \frac{\pi}{2}, \pi\right), \\
& D(0, \theta)=E\left(0, \frac{\pi}{2}, \theta\right) \frac{\partial^{2} \mathcal{S}_{I}}{\partial r^{2}}(0, \theta), \text { and } \\
& h_{1,2}^{\prime \prime}(0)=E\left(0, \frac{\pi}{2}, \pi\right) \int_{\frac{\pi}{2}}^{\pi} \frac{\partial^{2} \mathcal{S}_{I}}{\partial r^{2}}(0, \theta) E\left(0, \frac{\pi}{2}, \theta\right) d \theta .
\end{aligned}
$$

By using

$$
\begin{aligned}
& h_{1}^{\prime}(0)=h_{1,2}^{\prime}(0) \cdot h_{1,1}^{\prime}(0), \\
& h_{1}^{\prime \prime}(0)=h_{1,2}^{\prime \prime}(0) \cdot h_{1,1}^{\prime}(0)^{2}+h_{1,2}^{\prime}(0) \cdot h_{1,1}^{\prime \prime}(0),
\end{aligned}
$$

we get after applying several times the equality $E\left(0,0, \frac{\pi}{2}\right) \cdot E\left(0, \frac{\pi}{2}, \theta\right)=$ $E(0,0, \theta)$,

$$
\begin{aligned}
& h_{1}^{\prime}(0)=E(0,0, \pi), \\
& h_{1}^{\prime \prime}(0)=E(0,0, \pi) \int_{0}^{\pi} \frac{\partial^{2} \mathcal{S}_{I}}{\partial r^{2}}(0, \theta) E(0,0, \theta) d \theta .
\end{aligned}
$$

To evaluate $E(0,0, \pi)$, we write

$$
\begin{aligned}
\int_{0}^{\pi} \frac{t \sin ^{2} \theta d \theta}{1+t \sin \theta \cos \theta} & =\frac{1}{2} \int_{0}^{\pi} \frac{t d \theta}{1+t \sin \theta \cos \theta} \\
& -\frac{1}{2} \int_{0}^{\pi} \frac{t\left(\cos ^{2} \theta-\sin ^{2} \theta\right) d \theta}{1+t \sin \theta \cos \theta} \\
& =\frac{1}{2} \int_{0}^{\pi} \frac{t d \theta}{1+t \sin \theta \cos \theta}
\end{aligned}
$$


because the second integral has the primitive $\log (1+t \sin \theta \cos \theta)$ and it vanishes. Then, from (33) and (34) we get

$$
E(0,0, \pi)=\exp \left(-\frac{1}{2} \int_{0}^{\pi} \frac{t d \theta}{1+t \sin \theta \cos \theta}\right)=\exp \left(-\frac{\pi t}{\sqrt{4-t^{2}}}\right)
$$

Therefore, from (36) and (39) it follows (30).

Now, from (37) and using (33), (34) and (35), we see that

$$
\begin{aligned}
& h_{1}^{\prime \prime}(0)=-2 E(0,0, \pi) \\
& \quad \cdot \int_{0}^{\pi} \frac{b_{1}(\cos \theta+t \sin \theta)+b_{2} \sin \theta}{(1+t \sin \theta \cos \theta)^{2}} \exp \left(\int_{0}^{\theta} \frac{-t \sin ^{2} \phi d \phi}{1+t \sin \phi \cos \phi}\right) d \theta,
\end{aligned}
$$

and it can be shown (see the Appendix in Llibre and Ponce [15]) that

$$
h_{1}^{\prime \prime}(0)=-2 E(0,0, \pi)\left(b_{1} t+b_{2}\right)\left[1+\exp \left(-\frac{\pi t}{\sqrt{4-t^{2}}}\right)\right] .
$$

Hence, from (39) both (31) and (32) follow directly.

We write now a standard result that will also be used in the proof of Theorem 2.

Lemma 7. Consider for some $\delta>0$ sufficiently small a one-parameter family of $\mathcal{C}^{2}$ maps $\xi \mapsto h_{\mu}(\xi)$, with $|\mu|<\delta$ and $\xi \in \mathbf{R}$, having a fixed point at $\xi=0$, i.e., $h_{\mu}(0)=0$ for all $|\mu|<\delta$. Suppose that for $\mu=0$ this fixed point is non-hyperbolic; that is $h_{0}^{\prime}(0)=1$. If the conditions

$$
\left.\frac{d}{d \mu} h_{\mu}^{\prime}(0)\right|_{\mu=0} \neq 0, \text { and } h_{\mu}^{\prime \prime}(0) \neq 0
$$

are fulfilled, then the family of maps undergoes for $\mu=0$ a transcritical bifurcation at $\xi=0$; that is, apart from the fixed point $\xi=0$, there exists a curve $\xi(\mu)$ of fixed points in the $(\mu, \xi)$-plane passing through the origin of that plane and existing on both sides of $\mu=0$, giving rise to one additional fixed point for $\mu<0$ and for $\mu>0$. The slope of that curve at the origin is

$$
\frac{d \xi}{d \mu}(0)=-\frac{\left.\frac{d}{d \mu} h_{\mu}^{\prime}(0)\right|_{\mu=0}}{h_{\mu}^{\prime \prime}(0)}
$$

Proof: See for instance Wiggins [18, pp. 362-366]. 
Finally, we give the proof of Theorem 2 .

Proof of Theorem 2: We apply Lemma 7 to the Poincaré map $h_{1}$, see (29), taking $\mu=t$. From Lemma 6 we know that this map is nonhyperbolic at $\xi=0$ for $t=0$, and that

$$
\begin{aligned}
\left.\frac{d}{d t} h_{1}^{\prime}(0)\right|_{t=0} & =\frac{d}{d t}\left\{\exp \left(-\frac{\pi t}{\sqrt{4-t^{2}}}\right)\right\}_{t=0}=-\frac{\pi}{2} \neq 0, \\
\left.h_{1}^{\prime \prime}(0)\right|_{t=0} & =-4 b_{2} .
\end{aligned}
$$

Thus, from Lemma 7 , when $b_{2} \neq 0$, the map $h_{1}(\xi)$ undergoes for $t=0$ a transcritical bifurcation at $\xi=0$. From (26) and (17) statement (a) follows.

The additional curve of fixed points $\xi(t)$ has, at the origin of the plane $(t, \xi)$, the slope

$$
\frac{d \xi}{d t}(0)=-\frac{\pi}{8 b_{2}} .
$$

Therefore, since only the fixed points with $\xi>0$ correspond to periodic orbits of system (1)-(2), recalling again (26) and (17) the assertions of statements (b) and (c) (excepting the stability of limit cycles) are proved.

A local expression of $h_{1}(\xi)$ at $\xi=0$ gives

$$
h_{1}(\xi)-\xi=\left(h_{1}^{\prime}(0)-1\right) \xi+\frac{1}{2} h_{1}^{\prime \prime}(0) \xi^{2}+O\left(\xi^{3}\right) .
$$

Then, eliminating the root $\xi=0$ in the equation $h_{1}(\xi)-\xi=0$, we get that the other root $\xi(t) \neq 0$ satisfies

$$
h_{1}^{\prime}(0)-1+\frac{1}{2} h_{1}^{\prime \prime}(0) \xi(t) \approx 0,
$$

for $\xi(t)$ sufficiently small. That is,

$$
\xi(t) \approx 2 \frac{1-h_{1}^{\prime}(0)}{h_{1}^{\prime \prime}(0)} .
$$

Therefore, for $\xi(t)$ sufficiently small, we have

$$
h_{1}^{\prime}(\xi(t)) \approx h_{1}^{\prime}(0)+h_{1}^{\prime \prime}(0) \xi(t) \approx 2-h_{1}^{\prime}(0)=2-\exp \left(-\frac{\pi t}{\sqrt{4-t^{2}}}\right),
$$

where we have used Lemma 6 . So, $h_{1}^{\prime}(\xi(t))$ is greater than 1 if $t>0$ and less than 1 for $t<0$. Hence, recalling once more (26) and (17), the stability assertions of statements (b) and (c) are shown. 
From (40) and Lemma 6, we have

$$
\xi(t) \approx-\frac{1-\exp \left(-\frac{\pi t}{\sqrt{4-t^{2}}}\right)}{\left(b_{1} t+b_{2}\right) \exp \left(-\frac{\pi t}{\sqrt{4-t^{2}}}\right)\left[1+\exp \left(-\frac{\pi t}{\sqrt{4-t^{2}}}\right)\right]},
$$

and so the bifurcated limit cycle for system (27) is near the circle of radius $r=1 / \xi(t)$. Going backwards through (26), the bifurcated limit cycle for system (25) is near the circle of radius

$$
\frac{1}{r}=-\frac{1-\exp \left(-\frac{\pi t}{\sqrt{4 d-t^{2}}}\right)}{\left(b_{1} t+b_{2} d\right) \exp \left(-\frac{\pi t}{\sqrt{4 d-t^{2}}}\right)\left[1+\exp \left(-\frac{\pi t}{\sqrt{4 d-t^{2}}}\right)\right]} \sqrt{d}
$$

Statement (d) follows at once by using (17) and recalling the transformation (24), made to get (25) from (16).

Statement (e) comes from the fact that for $T=0$ and $t \neq 0$ the trace of system (27) has constant sign (see the standard Bendixson-Dulac criterion but adequately modified to continuous piecewise $\mathcal{C}^{1}$-systems, as in Proposition 3 of Llibre and Sotomayor [6]).

Statement (f) is a direct consequence of the hyperbolicity of the periodic orbit at infinity, see Lemma 6.

Acknowledgements. Both authors are supported by DGICYT grants numbers PB93-0860 and TAP94-0491 respectively.

\section{References}

1. S. Lefschetz, "Stability of non-linear control systems," Acad. Press, New York, 1965.

2. K. S. NAREndRa AND J. M. TAYLOR, "Frequency domain criteria for absolute stability," Acad. Press, New York, 1973.

3. J. N. Glover, Hopf bifurcation at infinity, Nonlinear Anal. 13 (1989), 1393-1398.

4. X. He, Hopf bifurcation at infinity with discontinuous nonlinearities, J. Austral. Math. Soc. Ser. B 31 (1991), 133-148. 
5. R. Lum AND L. O. ChuA, Global properties of continuous piecewise-linear vector fields. Part II: simplest symmetric case in $\mathbf{R}^{2}$, Memorandum UCB/ERL M90/29, University of California at Berkeley (1990).

6. J. Llibre And J. Sotomayor, Phase portrait of planar control systems, Nonlinear Anal. (to appear).

7. J. Llibre And E. Ponce, Global first harmonic bifurcation diagram for odd piecewise linear control systems, Dynam. Stability Systems 11 (1996), 49-88.

8. N. G. Lloyd, A note on the number of limit cycles in certain two-dimensional systems, J. London Math. Soc. 20 (1979), 277-286.

9. J. Sotomayor And R. PAterlini, Bifurcations of polynomial vector fields in the plane, in "Oscillation, Bifurcation and Chaos," (F. V. Atkinson, S. F. Langford and A. B. Mingarelli, eds.), CMS-AMS Conference Proceedings 8, Providence RI, 1987, pp. $665-685$.

10. T. R. Blows and C. Rousseau, Bifurcation at infinity in polynomial vector fields, J. Differential Equations 104 (1993), 215-242.

11. V. Guíñez, E. SÁEz AND I. SzÁntó, Simultaneous Hopf bifurcations at the origin and infinity for cubic systems, in "Dynamical Systems," (R. Bamon, R. Labarca and J. Palis, eds.), Pitman Research Notes in Mathematics 285, 1993, pp. 40-51.

12. W. L. Keith And R. H. RAnd, Dynamics of a system exhibiting the global bifurcation of a limit cycle at infinity, Internat. J. Non-Linear Mech. 20 (1985), 325-338.

13. L. Malaguti, Soluzioni periodiche dell'equazione di Liénard: biforcazione dall'infinito e non unicità, Rend. Istit. Mat. Univ. Trieste 19 (1987), 12-31.

14. M. Sabatini, Bifurcation from infinity, Rend. Sem. Mat. Univ. Padova 78 (1987), 237-253.

15. J. Llibre AND E. Ponce, Bifurcation of a periodic orbit from infinity in planar piecewise linear vector fields, Preprint (1996).

16. A. A. Andronov, E. A. Leontovich, I. I. Gordon And A. G. MAIER, "Qualitative Theory of Second-Order Dynamic Systems," John Wiley and Sons, New York-Toronto, 1973.

17. C.-T. Chen, "Linear System Theory and Design," Holt, Rinehart and Winston, Inc., 1984. 
18. S. Wiggins, "Introduction to Applied Nonlinear Dynamical Systems and Chaos," Text Applied Math. 2, Springer-Verlag, 1990.

Jaume Llibre:

Departament de Matemàtiques

Universitat Autònoma de Barcelona

08193 Bellaterra (Barcelona)

SPAIN

e-mail: jllibre@mat.uab.es
Enrique Ponce:

Departamento de Matemática Aplicada II

Escuela Superior de Ingenieros

Avda. Reina Mercedes

41012 Sevilla

SPAIN

e-mail: enrique@matinc.us.es

Rebut el 30 de Novembre de 1996 